\title{
A Comparison of Accuracy of Skeletal Maturity Indicators Using Cone-beam Computed Tomography, Lateral Cephalogram, and Hand-wrist Radiograph in Circumpubertal Dravidian Population
}

\author{
Munaif Vyshyambath ${ }^{1}$, Raghunath Nagasundara Rao ${ }^{2}$, Jyothikiran Hurkadle ${ }^{3}$
}

\begin{abstract}
Aims: The study aims to evaluate and compare cervical maturation stage using lateral cephalogram, cone-beam computed tomography (CBCT), and skeletal maturation using hand-wrist radiograph.

Materials and methods: Forty-eight patients of circumpubertal Dravidian population were chosen. Subjects were advised to take CBCT of cervical region, lateral cephalogram, and hand-wrist radiograph with adequate protective measures. The CBCT and lateral cephalogram were evaluated using the Lamparski index modified by Hassel and Farman as a reference to determine skeletal maturity using cervical vertebra. Hand-wrist radiograph was evaluated for different stages of maturation according to Fishman's index. Stages of maturity between hand-wrist radiograph, lateral cephalogram and CBCT were evaluated in circumpubertal male and female subjects for reliability and accuracy.

Results: When evaluating skeletal maturity indicator using these three diagnostic aids, it was found that all three were reliable with $p$ value $<0.05$ in all groups. The accuracy between $\mathrm{CBCT}$, lateral cephalogram, and hand-wrist shows skeletal maturity indicator using CBCT was highly accurate than lateral cephalogram and hand-wrist radiographs.

Conclusion: Cervical vertebrae maturation index (CVMI) using lateral cephalogram and CBCT shows high reliability in prepubertal, pubertal, and post-pubertal male and female subjects. The skeletal maturity indicator using hand-wrist shows reliable tool for skeletal maturity. When the accuracy between the aids was compared, it revealed that CBCT is the most accurate diagnostic aid in assessing skeletal maturity indicator. Clinical significance: The results of this study suggest that CBCT can be used to assess skeletal maturity and, therefore, we can avoid an additional radiogram (hand-wrist radiography) whenever $\mathrm{CBCT}$ is considered as an investigating tool for orthodontic patients.

Keywords: Cone beam computed tomography, Hand-wrist, Lateral cephalogram, Skeletal maturation.

World Journal of Dentistry (2020): 10.5005/jp-journals-10015-1705
\end{abstract}

\section{INTRODUCTION}

Growth is defined as changes in morphology which is a fathomable parameter. It is a composite of morphogenetic and histogenetic changes as a response to environmental influences and genetics. Growth in human is characterized by significant changes in the rate of progression of different individuals toward physiologic maturity. It is one of the most contrasting variations in nature with a remarkable role in the etiology, evaluation of diagnosis, treatment planning, retention, and stability of any cases of malocclusion. ${ }^{1}$

Various methods are used to evaluate skeletal maturity. They include the use of hand-wrist radiographs, cervical vertebrae maturation index (CVMI) from lateral cephalograms, midpalatal suture maturation using occlusal radiographs, and third molar and canine mineralization using orthopantomograms to determine dental age. Till date, hand-wrist radiography has been used to evaluate skeletal maturation, similar to a comprehensive pattern of the subject's growth to a preset pattern of reference. Skeletal maturation is commonly identified by evaluating the events of ossification of hand-wrist bones due to the considerable number of different types of smaller bones present in those areas.

The maturity of skeleton and its desirable relationship to growth of the face is evaluated using the cervical vertebrae morphology. Lamparski suggested a number of standards for CVMI. The efficiency of lateral cephalometric radiography to evaluate maturity
${ }^{1-3}$ Department of Orthodontics, JSS Dental College and Hospital, JSS Academy of Higher Education and Research, Mysuru, Karnataka, India Corresponding Author: Munaif Vyshyambath, Department of Orthodontics, JSS Dental College and Hospital, JSS Academy of Higher Education and Research, Mysuru, Karnataka, India, Phone: +91 7349456653, e-mail: Munaifusman@gmail.com

How to cite this article: Vyshyambath M, Rao RN, Hurkadle J. A Comparison of Accuracy of Skeletal Maturity Indicators Using Conebeam Computed Tomography, Lateral Cephalogram, and Hand-wrist Radiograph in Circumpubertal Dravidian Population. World J Dent 2020;11(2):139-145.

Source of support: Nil

Conflict of interest: None

of the skeleton has been looked into, and data suggests that the cervical vertebrae may be optimal indicators of skeletal maturity. ${ }^{2}$ Arrival of cone beam computed tomography (CBCT) has revealed newer avenues and significantly improved the accuracy of diagnosis and treatment planning in growing patients. The pictures of head and neck are obtained in multiple dimensions, including cervical vertebrae, with the utility of CBCT.

The application of CBCT in orthodontics is relevant as it allows more complete and detailed evaluation of craniofacial anatomy in some cases. The request of a CBCT examination may

(c) The Author(s). 2020 Open Access This article is distributed under the terms of the Creative Commons Attribution 4.0 International License (https://creativecommons. org/licenses/by-nc/4.0/), which permits unrestricted use, distribution, and non-commercial reproduction in any medium, provided you give appropriate credit to the original author(s) and the source, provide a link to the Creative Commons license, and indicate if changes were made. The Creative Commons Public Domain Dedication waiver (http://creativecommons.org/publicdomain/zero/1.0/) applies to the data made available in this article, unless otherwise stated. 
provide an assembly of data that allow exhibition of images in sections (axial, coronal, and sagittal) and by three-dimensional (3D) reconstructions, which are very didactic and may even be morphologically more expressive than the images of isolated sections. Professionals in charge of a CBCT machine or requesting the $\mathrm{CBCT}$ examination must provide information on the possible findings based on complete interpretation of images achieved. Based on the $3 D$ analysis of the cervical vertebrae, he potential utilization of $C B C T$ for evaluation of skeletal maturation has been suggested. ${ }^{3}$

Consequently, there is a need to establish a correlation and reliability between hand-wrist radiographs and CVMI using lateral cephalogram and CBCT. Aim of the present study was to evaluate and compare the accuracy of skeletal maturity indicator using $C B C T$, lateral cephalogram, and hand-wrist radiograph in circumpubertal Dravidian population.

\section{Materials and Methods}

Forty-eight subjects of Dravidian ethnic origin reporting to the Department of Orthodontics, JSS Dental College and Hospital, Mysuru, were included in the study. The participants were divided as follows: males of 10-18 years, and they were further divided into group I ( $\geq 10$ to $\leq 13$ years), group II ( $>13$ to $\leq 15$ years), and group III ( $>15$ to $\leq 18$ years); female subjects of 8 to 16 years, who were further divided into group I ( $\geq 8$ to $\leq 11$ years), group II ( $>11$ to $\leq 13$ years), and group III ( $>13$ to $\leq 16$ years). Informed consent was obtained from the participants.

\section{Selection Criteria}

Inclusion criteria for selection are patients in the circumpubertal age-group, female subjects in the age range of 8-16 years, and male subjects in the age range of 10-18 years. Patient with systemic illness, growth abnormalities, bleeding disorders, and chronic medications were excluded from the study.

The study protocol was approved by the Institutional Ethical Committee of JSS Dental College and Hospital, Mysore, and JSS Academy of Higher Education and Research.

Stratified random sampling was employed to select the required sample. The subjects who satisfied the inclusion criteria and agreed to participate were included in the study.

\section{Methodology}

\section{Materials for Radiographs}

Lateral cephalograms to evaluate CVMI was taken using panoramic radiographic machine- "PLANMECA SCARA-3" (promax SW 1.20.1.0.R, Proline XCGUI 1.4.0.0.1 PK 5.60.pg5.60, Helsinki, Finland) and processed using direct digital processing unit_-charged couple device containing analog-to-digital converter located within or connected to the computer.

Hand-wrist radiograph for recording maturational status was taken using panoramic radiographic machine-Rotograph 230 eur (Villa Systemi Medicali, Italy) with provision for automatic exposure parameters with a magnification of $1 \mathrm{~T}$ mat green-sensitive films (Eastman Kodak, Rochester, New York, USA), X-ray machine radiographic film cassette $-15 \times 30 \mathrm{~cm}$ (Konica, Tokyo Japan) with green-sensitive intensifying screens (Konica Minolta KR-II, Tokyo Japan), and processed with automatic extraoral radiographic film processor-Promax 5 Speed, with time and temperature controls with auto-replenishing (Chayagraphics, India), and fresh processing chemicals-developer and fixer (Kodak automatic processing chemicals, New York, USA).

The CBCT of cervical vertebra was taken with CS9300 machine using the parameters of $90 \mathrm{kV} 5 \mathrm{Ma} 8.01$ seconds. The unit has three field-of-view (FOV) modes, namely, facial (F), panoramic (P), and implant (I) modes. The size of the FOV and voxels for each mode is, respectively, $192.2 \mathrm{~mm}$ in diameter and 0.36 minutes in $\mathrm{F}$ mode, $150 \mathrm{~mm}$ in diameter and $0.293 \mathrm{~mm}$ in $\mathrm{P}$ mode, and $102.4 \mathrm{~mm}$ in diameter and $0.200 \mathrm{~mm}$ in I mode. Images from F and P modes only will be selected. The CBCT will be evaluated using "CS9300 Select" software on the computer (Fig. 1).

\section{Method for Interpretation of Radiographs and CBCT}

The radiographs and $C B C T$ were interpreted by three investigators. Case history regarding age, sex, pubertal status, history of accidents or previous trauma to cervical vertebrae or upper limb, or other disorders was recorded. The CBCT was evaluated using CS9300 Select software on the computer using the Lamparski modified by Hassel and Farman method of determination of skeletal maturity using cervical vertebra as a reference (Fig. 2). After retrieval of the lateral cephalogram film, the CVMI was evaluated according to Hassel and Farman's method, which described the stages according to the morphological characteristics of the second, third, and fourth vertebrae (Fig. 3). Hand-wrist radiograph was taken and evaluated for different stages of maturation according to Fishman's index (Fig. 4 and Table 1). Stages of maturity between hand-wrist radiograph and lateral cephalogram, hand-wrist and $C B C T$, and lateral cephalogram and CBCT were evaluated in circumpubertal male and female subjects.

\section{Statistics}

In the study, diagnosis values are considered in terms of ordinal scale; therefore, nonparametric test was performed. Descriptive statistics median and mean ranks are computed. Kruskal-Wallis $(H)$ test is carried out to see the significant difference across the groups $\left(G_{1}, G_{2}\right.$, and $\left.G_{3}\right)$. Further, Mann-Whitney $U$ test was carried out to see the pairwise significant difference between groups $\left(G_{1} v s G_{2}\right)$, $\left(G_{1} v s G_{3}\right)$, and $\left(G_{2} v s G_{3}\right)$. Also, Mann-Whitney $U$ test (nonparametric test) has been carried out to understand the significant difference between genders (male vs female). The one-way analysis of variance (ANOVA) is used to measure a quantitative dependent variable by a single-factor (independent) variable. Analysis of variance is used to test the hypothesis that several means are equal. This technique is an extension of the two-sample $t$ test.

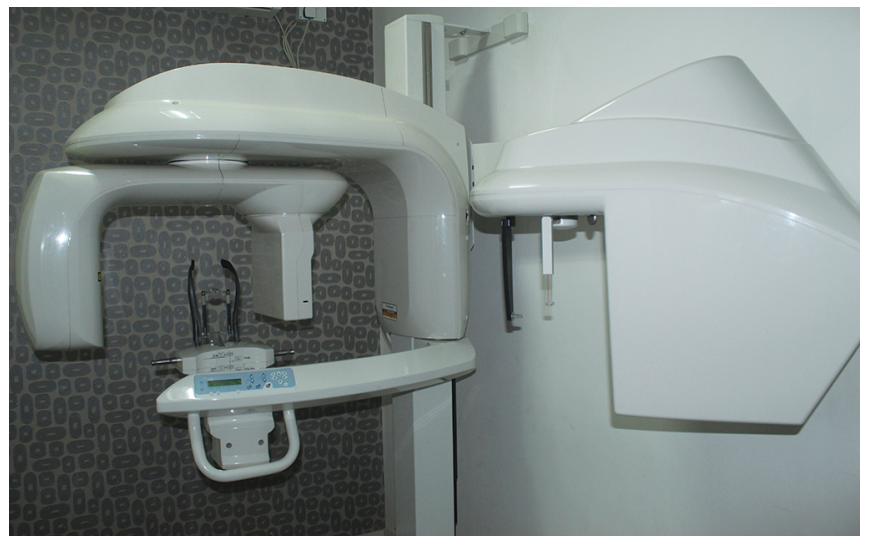

Fig. 1: Cone-beam computed tomography machine 


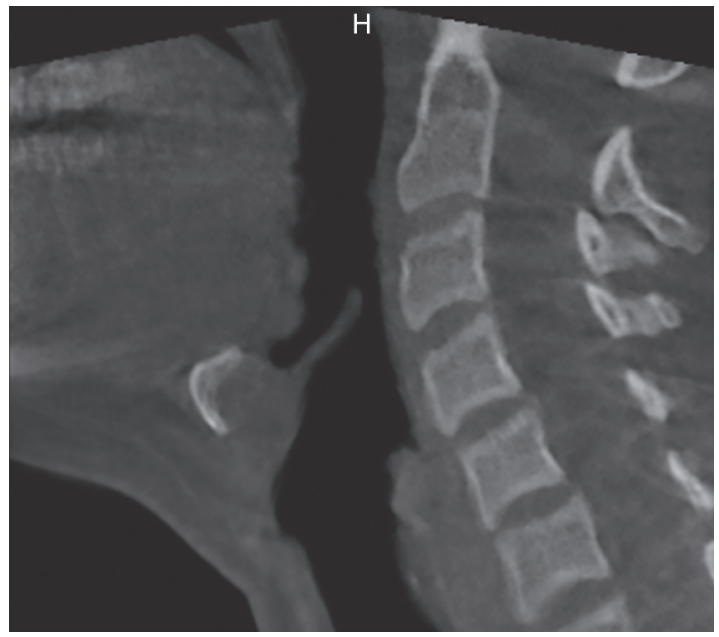

Fig. 2: Cone-beam computed tomography image of cervical vertebra of group II subject

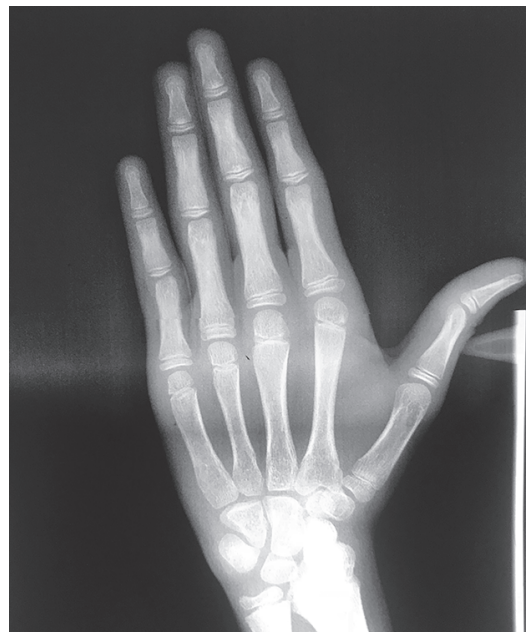

Fig. 4: Hand-wrist radiograph of group I subject

\section{Results}

Descriptive statistics was used to compare the data between the groups (Fig. 5 and Table 2).

The mean rank for lateral cephalogram (D1) in groups I, II, and III was $11.59,23$, and 38.91, respectively. The mean rank for hand-wrist radiograph (D2) in group II was $11.41,23.75$, and 38.34, respectively, and the mean rank for CBCT (D3) in group III was 11.53, 23.25, and 38.72 , respectively.

Nonparametric tests of significance was used to analyze the data and the results revealed a statistically significant difference between males across groups I, II, and III, with respect to all three diagnostic aids [lateral cephalogram (D1), hand-wrist (D2), and CBCT (D3)] (Fig. 6 and Table 3). Nonparametric tests of significance was used to analyze the data and the results revealed a statistically significant difference between females across groups I, II, and III, with respect to all three diagnostic aids (D1, D2, and D3) (Fig. 7 and Table 4). The results show that all three diagnostic aids are reliable to check for skeletal maturity.

One-way ANOVA was performed to check the significance between the three diagnostic aids. The results revealed that D3 showed higher significance in comparison to D1 and D2. The test

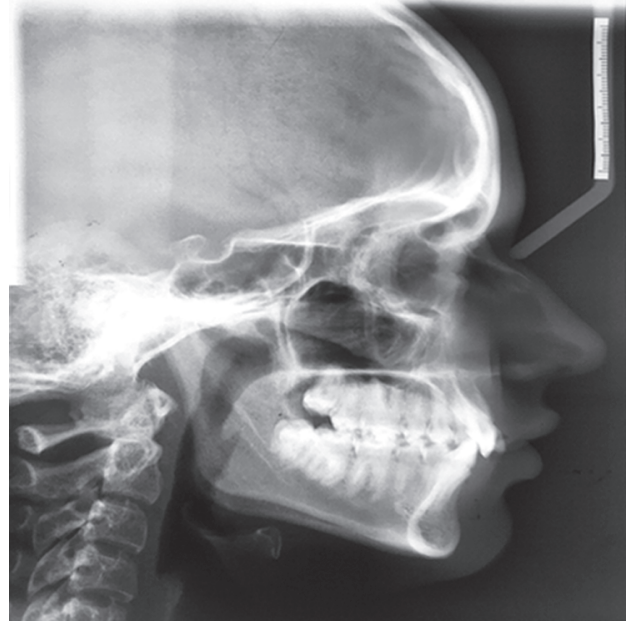

Fig. 3: Lateral cephalogram of group II subject

Table 1: Comparison between D1, D2, D3

\begin{tabular}{lccll}
\hline \multicolumn{2}{l}{$\begin{array}{l}\text { Comparison between } \\
\text { groups }\end{array}$} & $\begin{array}{l}\text { Mean } \\
\text { difference }(I-J)\end{array}$ & Std. error & Sig. \\
\hline D1 & D2 & $-1.87500^{*}$ & 0.49042 & 0.000 \\
& D3 & 0.22917 & 0.49042 & 0.641 \\
D2 & D1 & $1.87500^{*}$ & 0.49042 & 0.000 \\
& D3 & $2.10417^{*}$ & 0.49042 & 0.000 \\
D3 & D1 & -0.22917 & 0.49042 & 0.641 \\
& D2 & $-2.10417^{*}$ & 0.49042 & 0.000 \\
\hline
\end{tabular}

Note: ${ }^{*}=$ significant at $p<0.05$, lateral cephalogram (D1), hand-wrist (D2), and $\mathrm{CBCT}(\mathrm{D} 3)$

also revealed no significant difference between D1 and D3. Thus, it can be concluded that D3 is more accurate in comparison with D1 and D2 (Table 5). The results of the present study show that CBCT is a more accurate diagnostic tool for assessing skeletal maturity in comparison to lateral cephalogram and hand-wrist radiographs.

\section{Discussion}

As orthodontics involves study of growth, growth assessment is the process of assessing the maturational status of an individual. Skeletal maturity indicators help to give as much information as possible on the growth pattern and the degree of accomplishment of that pattern for each patient.

The success of many dental procedures, including orthodontic treatment and orthognathic surgery, is highly relied on the determination of skeletal maturity in patients. ${ }^{1}$ Residual bone growth may interfere with certain procedures, while other procedures work best in patients who are still experiencing bone growth. Accordingly, the accurate finding of skeletal maturity plays an important role in the timing of many dental procedures. There are a large number of growth assessment methods as suggested by many authors but the main concern is about the reliability. The present condition in clinical orthodontics requires an accurate method with well-defined and easily understandable stages that could be interpreted in a cross-sectional study without requiring long observation periods.

Simpson and Raper explained the importance of using hand-wrist radiographs as an important adjunct to diagnosis 


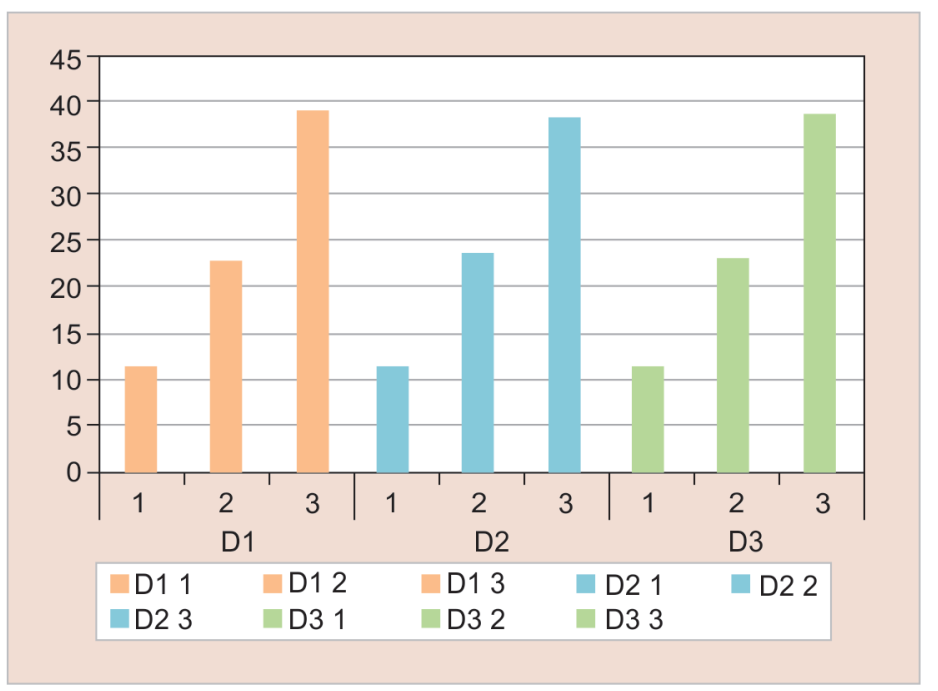

Fig. 5: Descriptive statistics between groups I, II, and III across D1, D2, and D3

Table 2: Descriptive statistics between groups I, II, and III across D1, D2, and D3

\begin{tabular}{lllll}
\hline & Groups & $n$ & Mean rank & Median \\
\hline D1 & 1 & 16 & 11.59 & 2.00 \\
& 2 & 16 & 23.00 & 2.00 \\
& 3 & 16 & 38.91 & 2.00 \\
D2 & 1 & 16 & 11.41 & 3.00 \\
& 2 & 16 & 23.75 & 4.00 \\
& 3 & 16 & 38.34 & 2.50 \\
D3 & 1 & 16 & 11.53 & 5.50 \\
& 2 & 16 & 23.25 & 9.00 \\
& 3 & 16 & 38.72 & 6.00 \\
\hline
\end{tabular}

Lateral cephalogram (D1), hand-wrist (D2), and CBCT (D3)

in orthodontics. Crater and Howard reported radiographic significance in carpel bones of children. Hellman published his observation on the ossification of epiphyseal cartilages of the hand. Although it is necessary to take a radiograph of the entire hand and wrist area, not all ossification stages are used simultaneously at any time throughout the growth period. During early childhood, carpals can be used; and during late childhood and puberty, the changes occurring in the metacarpal and phalanges provide better information. Apart from the phalanx of fingers, the adductor sesamoid of the metacarpal joint of the first finger, the pisiform, and hook of hamate are the key indicators of hand-wrist radiograph.

Cervical vertebrae are used as a reliable method of skeletal growth prediction and biological maturity indicator. The size and shape of cervical vertebrae in growing subjects have gained increasing interest as a biological indicator in individual skeletal maturity. The main reason of rising popularity of this method is that the analysis of cervical vertebrae maturation (CVM) is performed on the lateral radiograph of patient's head which is the type of film used routinely in orthodontic diagnosis to analyze the skeletal morphology and directional growth patterns. Since properly utilized cervical vertebrae provide a reliable assessment of pubertal growth spurt, it will be a beneficial process to use these films with this purpose too, as the number of exposures is decreased. ${ }^{4}$

The result of recent advances in the field of radiology, CBCT is becoming more popular as an investigating tool for orthodontic patients. We can see the images of head and neck in all three planes, including cervical vertebrae, using CBCT. Visualization of individual sagittal slices of a 3D structure is a feature in $C B C T$ imaging. These slices are distinguished from lateral cephalometric radiographs and other $2 \mathrm{D}$ radiographs though they appear similar in their portrayal of cervical spine. They are derived from a 3D scan and, thus, preserve $3 \mathrm{D}$ qualities. This factor explains the varying degrees of concavity of lower borders of second to fourth cervical vertebrae and shape of the bodies of third and fourth cervical vertebrae, which are key maturational indicators of various CVM methods.

The application of CBCT in orthodontics is relevant because it allows more complete and detailed evaluation of craniofacial anatomy in some cases. The request of a CBCT examination may provide an assembly of data that allows exhibition of images in sections (axial, coronal, and sagittal) and by 3D reconstructions, which are very didactic and may even be morphologically more expressive than images of isolated sections. The potential utilization of CBCT for evaluation of skeletal maturation has been suggested by $3 \mathrm{D}$ analysis of the cervical vertebrae. ${ }^{5}$

Additionally, the radiographic artifacts associated with lateral cepholagrams justify the need to look at CBCT-CVMI method to assess growth since it is a $3 \mathrm{D}$ radiograph, and there will not be any fogging in $\mathrm{CBCT}$. Hence, it is a valuable tool in the determination of skeletal age maturation.

The study was designed with a purpose to equip the orthodontist with a new evaluation tool which would enable the orthodontist to accurately evaluate the amount of growth remaining, using existing growth assessment methods in a single clinical visit and using a single diagnostic tool.

The results of the present study are discussed under the following headings.

\section{To Evaluate Cervical Maturation Stage Using Lateral Cephalogram and CBCT}

The skeletal maturational indicator using lateral cephalogram and CBCT was performed in all three circumpubertal age-groups and in males and females. Results revealed a statistically significant difference within all groups and genders except for D1 (lateral cephalogram) when compared across groups I and II which showed a statistically insignificant difference with a $p$ value $<0.05$ (Table 3). 


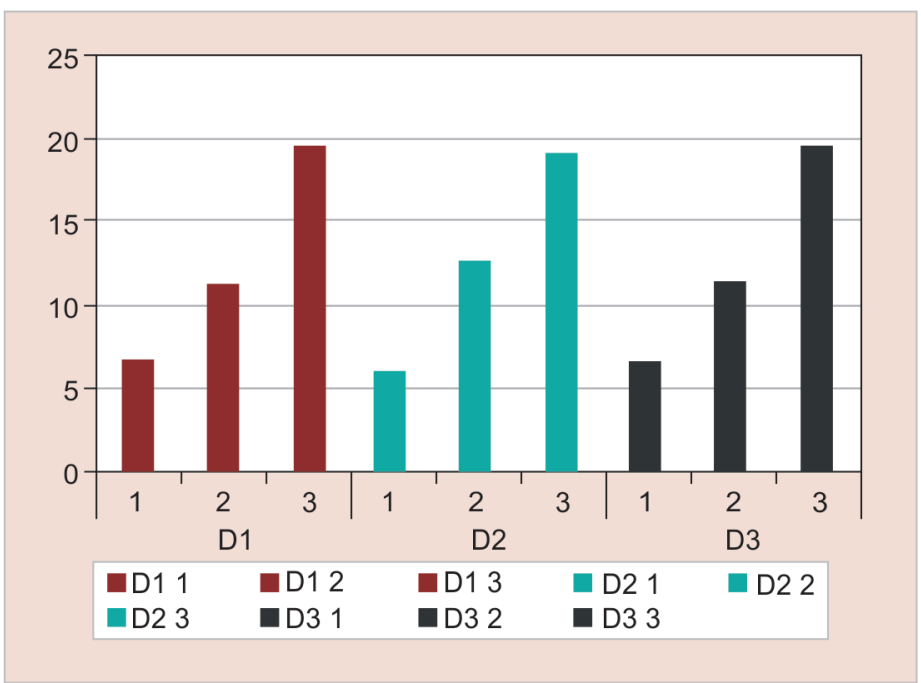

Fig. 6: Comparison of D1, D2, and D3 among males across groups I, II, and III

Table 3: Comparison of D1, D2, and D3 of males across groups I, II, and III

\begin{tabular}{|c|c|c|c|c|c|c|}
\hline & Groups & $n$ & Mean rank & Chi-square & $d f$ & $p$ value \\
\hline \multirow[t]{3}{*}{ D1 } & 1 & 8 & 6.69 & 15.198 & 2 & $0.001^{*}$ \\
\hline & 2 & 8 & 11.19 & & & \\
\hline & 3 & 8 & 19.63 & & & \\
\hline \multirow[t]{3}{*}{ D2 } & 1 & 8 & 5.94 & 13.967 & 2 & 0.001 \\
\hline & 2 & 8 & 12.63 & & & \\
\hline & 3 & 8 & 18.94 & & & \\
\hline \multirow[t]{3}{*}{ D3 } & 1 & 8 & 6.56 & 14.745 & 2 & 0.001 \\
\hline & 2 & 8 & 11.44 & & & \\
\hline & 3 & 8 & 19.50 & & & \\
\hline
\end{tabular}

Note: ${ }^{*}=$ significant at $p<0.05$, lateral cephalogram (D1), hand-wrist (D2), and CBCT (D3)

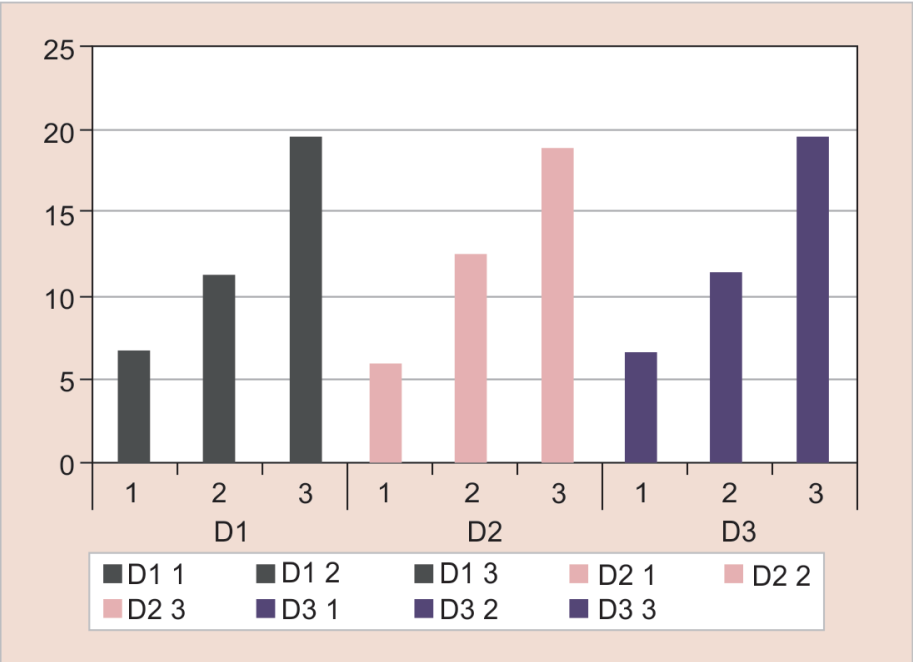

Fig. 7: Comparison of diagnostic aids among females in all groups

A study aimed to investigate the relationship of the stages of CVM to growth changes in the mandible by O'Reilly et al. ${ }^{6}$ suggested that the stages of CVM were related to mandibular growth changes taking place during puberty. The vertebral stages 1 through 3 occurred in the accelerative growth phase, with stages 2 and 3 occurring most frequently in the year preceding the maximum increment of mandibular growth. Stages 4 through 6 were observed to occur during the decelerative phase of growth after peak velocity. Thus, cervical vertebral stages of maturation could be utilized to assess the timing of mandibular growth and changes at puberty. In 
Table 4: Comparison of D1, D2, and D3 among females across groups I, II, and III

\begin{tabular}{|c|c|c|c|c|c|c|}
\hline & Groups & $n$ & Mean rank & Chi-square & $d f$ & $p$ value \\
\hline \multirow[t]{3}{*}{ D1 } & 1 & 8 & 6.69 & 15.929 & 2 & $0.001^{*}$ \\
\hline & 2 & 8 & 11.19 & & & \\
\hline & 3 & 8 & 19.63 & & & \\
\hline \multirow[t]{3}{*}{ D2 } & 1 & 8 & 5.94 & 16.804 & 2 & 0.001 \\
\hline & 2 & 8 & 12.63 & & & \\
\hline & 3 & 8 & 18.94 & & & \\
\hline \multirow[t]{3}{*}{ D3 } & 1 & 8 & 6.56 & 16.658 & 2 & 0.001 \\
\hline & 2 & 8 & 11.44 & & & \\
\hline & 3 & 8 & 19.50 & & & \\
\hline
\end{tabular}

Note: ${ }^{*}=$ significant at $p<0.05$, lateral cephalogram (D1), hand-wrist (D2), and CBCT (D3)

Table 5: Analysis of variance

\begin{tabular}{llllll}
\hline & Sum of squares & $d f$ & Mean square & $F$ & Sig. \\
\hline Between groups & 127.931 & 2 & 63.965 & 11.081 & 0.000 \\
Within groups & 813.896 & 141 & 5.772 & & \\
Total & 941.826 & 143 & & & \\
\hline
\end{tabular}

the current study, both CBCT and lateral cephalogram used CVMI for assessing skeletal maturity and give us reliable results. So the current study is in accordance with the previous study conducted for the assessment of skeletal maturity.

Baccetti et al. ${ }^{2}$ studied CVM method for assessing optimal treatment timing in dentofacial orthopedics and proposed that the CVM method enables the clinician to identify optimal time for the treatment of a series of dentoskeletal disharmonies in all three planes of space. This is in accordance with the results obtained in the present study.

A study on geometric morphometric evaluation of cervical vertebrae shape and its relationship to skeletal maturation by Chatzigiannia et al. ${ }^{7}$ suggested that vertebral shape is strongly correlated to skeletal age but does not offer better predictive value than chronological age. These studies help to correlate the results of the current study.

Similar findings were reported by Joshi et al. in the assessment of $\mathrm{CBCT}$ as a cervical maturation indicator, suggesting that CVM assessment with CBCT provides reliable assessment of pubertal growth spurt, ${ }^{8}$ and, therefore, this is in consonance with the current study that $\mathrm{CBCT}$ can be used to assess skeletal maturity.

Byun et al. conducted a study of quantitative assessment of CVM using CBCT and proposed that CBCT-CVMI analysis can be used to estimate skeletal maturation status. ${ }^{3}$ In agreement to the previous study, the current study evaluated CBCT-CVMI as a reliable diagnostic aid for skeletal maturity indicators.

A study conducted on 3D reconstruction of individual cervical vertebrae from $C B C T$ images by Shi et al. suggested that segmentation of individual vertebra is possible from CBCT volumetric data sets. ${ }^{9}$ The results of the current study reveal that CBCT is a valuable tool to assess skeletal maturity. This is in agreement with the previous studies.

\section{To Evaluate Skeletal Maturation Using Hand-wrist Radiograph}

The skeletal maturation indicator using hand-wrist was performed in all three circumpubertal age-groups and in males and females. Results revealed a statistically significant difference within all groups and genders, with a $p$ value $<0.05$ (Table 3 ). Hence, the findings suggest that hand-wrist is also a valuable tool in evaluating skeletal maturation.
Simpson et al. explained the importance of hand-wrist as a skeletal maturity indicator in orthodontics followed by Crater et al. who suggested radiographic significance in carpel bones of children.

Fishman gave a system for evaluating skeletal maturity from hand-wrist radiographs with complete details for implementation in clinical practice. His study demonstrated that hand-wrist radiographs could provide information on growth and maturation status with clinically important applications in dentofacial diagnosis and therapy. ${ }^{10}$ The current study results showed that handwrist is a reliable diagnostic aid as skeletal maturity indicator in circumpubertal age-groups of Dravidian population.

Sidlauskas et al. conducted a study to establish the relationship between the hand-wrist maturation stage, maturation level, and growth changes in mandible, and they suggested that maturational stages and levels demonstrated close correlation with maxillary and mandibular growth velocity. ${ }^{11}$ The present study was conducted on the subjects of circumpubertal age-groups where the growth of maxilla and mandible occurs. Hence, the results of the present study can be correlated with the results obtained from the previous study.

In a study conducted by Grave and Brown ${ }^{12}$ it was proposed that the peak growth velocity of stature in relation to a more extensive series of ossification events took place in the hand and wrist skeleton around the time of puberty. The results of the present study illustrate that hand-wrist radiograph is a valuable tool to assess skeletal maturity. This is in agreement with previous studies when all three diagnostic aids were compared with respect to gender, and it revealed a statistically insignificant difference ( $p$ value $>0.05$ ) (Table 4)

\section{To Compare Skeletal Maturation Using Lateral Cephalogram, Hand-wrist, and CBCT}

The one-way ANOVA revealed that CBCT-CVMI had a higher statistical significance ( $p$ value $<0.05$ ) in comparison to lateral cephalogram and hand-wrist radiograph. The test also revealed no statistical significant difference between lateral cephalogram and CBCT-CVMI. Thus, it can be derived from the results that CBCT-CVMI is more accurate in comparison with lateral cephalogram and hand-wrist.

A study by Alkhal et al. to correlate chronological age, CVM, and Fishman's hand-wrist skeletal maturity indicators suggested 
that CVM is a valid indicator of skeletal growth during the circumpubertal age-group and has a high correlation with the hand wrist maturation (HWM) ${ }^{13}$ In the present study, lateral cephalogram showed statistically significant correlation compared to hand-wrist radiograph ( $p \leq 0.05)$.

A study of comparative evaluation of skeletal maturity indicators - hand-wrist bone analysis and cervical vertebral analysis conducted by Gandini et al. proposed that vertebral analysis on a lateral cephalogram is as valid as hand-wrist bone analysis and has the advantage of reducing the radiation exposure of growing subjects. ${ }^{14}$ The results of the present study shows that skeletal maturity indicators using hand-wrist radiograph is as reliable as CVM using lateral cephalogram.

The present study evaluates the accuracy and reliability of CVMI-CBCT and CVMI-lateral cephalogram and the results show that CBCT is reliable and accurate in prediction of skeletal maturity. This is in compliance with the study by Choi et al. ${ }^{15}$ on cervical vertebral body's volume as a parameter for predicting the skeletal maturation stages. They used volumetric CBCT images of cervical vertebra and hand-wrist radiograph and proposed a new formula for predicting skeletal maturation by using regression analysis and the results suggest that volumetric parameters of cervical vertebrae measured using CBCT are useful in regression models. The derived regression model has the potential for clinical application as it enables a simple and quantitative analysis to evaluate skeletal maturation level.

A previous study by Bruno et al. to compare the accuracy and reliability of craniometric measurements on lateral cephalometry and $3 \mathrm{D}$ measurements on CBCT scans advocated that $\mathrm{CBCT}$ craniometric magnitudes are accurate to a subvoxel size and possibly can be used as a measurable orthodontic aid for diagnosis. The 2D cephalometric standards cannot be readily used for 3D because of the differences in measurement accuracy between the examinations. ${ }^{16}$ The current study compared the reliability and accuracy of three diagnostic aids, namely, $\mathrm{CBCT}$, hand-wrist, and lateral cephalogram, it showed that CBCT has higher statistical significance compared to other diagnostic aids ( $p$ value $<0.05$; Table 5 ).

\section{Limitations of the Study}

Further studies have to be conducted in a larger population. Also, the diagnostic tools such as $C B C T$ is expensive compared to other tools, which makes it a limitation of the study.

\section{Conclusion}

From the results of the present study it can be concluded that CVMI using lateral cephalogram and CBCT show high reliability in circumpubertal male and female subjects. Skeletal maturity indicator using hand-wrist radiographs shows high reliability in circumpubertal male and female subjects. The CBCT has the highest accuracy when compared to hand-wrist and lateral cephalogram for all groups and both genders.

\section{Clinical Significance}

The results of this study suggest that CBCT can be used to assess skeletal maturity; and, therefore, we can avoid an additional radiogram (hand-wrist radiography) whenever $\mathrm{CBCT}$ is considered as an investigating tool for orthodontic patients.

\section{References}

1. Todd TW. Atlas of skeletal maturation. Part 1, hand. London, United Kingdom: Kimpton; 1937.

2. Baccetti T, Franchi L, McNamara Jr JA. The cervical vertebral maturation (CVM) method for the assessment of optimal treatment timing in dentofacial orthopedics. Semin Orthod 2005;11(3):119-129. DOI: 10.1053/j.sodo.2005.04.005.

3. Byun B-R, Kim Y-I, Yamaguchi T, et al. Quantitative assessment of cervical vertebral maturation using cone beam computed tomography in Korean girls. Comput Math Methods Med 2015;2015:405912. DOI: 10.1155/2015/405912.

4. Bonfim MAE, Costa ALF, Fuziy A, et al. A study of cervical vertebrae maturation index estimates on cone beam $C T$ : $3 D$ reconstructions vs sagittal sections. Dentomaxillofac Radiol 2016;45:20150162. DOI: 10.1259/dmfr.20150162.

5. Cho S-M, Hwang C-J. Skeletal maturation evaluation using mandibular third molar development in adolescents. Korean J Orthod 2009;39(2):120-129. DOI: 10.4041/kjod.2009.39.2.120.

6. O'Reilly MT,YannielloGJ. Mandibulargrowth changesandmaturation of cervical vertebrae--a longitudinal cephalometric study. Angle Orthod 1988;58(2):179-184. DOI: 10.1043/0003-3219(1988)058<0179:MGCAM O>2.0.CO;2.

7. Chatzigiannia A, Halazonetis DJ. Geometric morphometric evaluation of cervical vertebrae shape and its relationship to skeletal maturation. Am J Orthod Dentofacial Orthop 2009;136(4):481.e1-481.e9 10.1016/j. ajodo.2009.04.017.

8. Joshi V, Yamaguchi T, Matsuda Y, et al. Skeletal maturity assessment with the use of cone-beam computerized tomography. Oral Surg Oral Med Oral Pathol Oral Radiol 2012;113(6):841-849. DOI: 10.1016/j. oooo.2011.11.018.

9. Shi H, Scarfe WC, Farmanc AG. Three-dimensional reconstruction of individual cervical vertebrae from cone-beam computed-tomography images. Am J Orthod Dentofacial Orthop 2007;131(3):426-432. DOI: 10.1016/j.ajodo.2005.12.031.

10. Fishman LS. Radiographic evaluation of skeletal maturation, a clinically oriented method based on hand-wrist films. Angle Orthod 1982;52(2):88-112. DOI: 10.1043/0003-3219(1982)052<0088:REOSM> 2.0.CO;2.

11. Sidlauskas A, Zilinskaite L, Svalkauskiene V. Mandibular pubertal growth spurt prediction. Part one: method based on the hand-wrist radiographs. Stomatologija 2005;7(1):16-20.

12. Grave KC, Brown T. Skeletal ossification and the adolescent growth spurt. Am J Orthod Dentofacial Orthop 1976;69(6):611-619. DOI: 10.1016/0002-9416(76)90143-3.

13. Alkhal HA, Wong RWK, Rabie ABM. Correlation between chronological age, cervical vertebral maturation and fishman's skeletal maturity indicators in Southern Chinese. Angle Orthod 2008;78(4):591-596. DOI: 10.2319/0003-3219(2008)078[0591:CBCACV]2.0.CO;2.

14. Gandini P, Mancini M, Andreani F. A comparison of hand-wrist bone and cervical vertebral analyses in measuring skeletal maturation. Angle Orthod 2006;76(6):984-989. DOI: 10.2319/070605-217.

15. Choi Y-K, Kim J, Yamaguchi T. Cervical vertebral body's volume as a new parameter for predicting the skeletal maturation stages. Biomed Res Int 2016;2016:8696735. DOI: 10.1155/2016/8696735.

16. Gribel BF, Gribel MN, Frazäo DC, et al. Accuracy and reliability of craniometric measurements on lateral cephalometry and $3 D$ measurements on CBCT scans. Angle Orthod 2011;81(1):26-35. DOI: 10.2319/032210-166.1. 